\title{
Modification of Membrane Surface using ALD ZnO for Anti-Biofouling
}

\author{
Kang-Hee Park ${ }^{1}$, Beom Joon Kim ${ }^{1}$, Gwon Deok Han², Joon Hyung Shim ${ }^{2}$, Hee-Deung Park ${ }^{1}$ \\ ${ }^{1}$ School of Civil, Environmental and Architectural Engineering/Korea University \\ 145, Anam-Ro, Seongbuk-gu, Seoul 02841, South Korea \\ newpkh@korea.ac.kr; goodmanner778@naver.com; heedeung@korea.ac.kr \\ ${ }^{2}$ School of Mechanical Engineering /Korea University \\ 145, Anam-Ro, Seongbuk-gu, Seoul, South Korea \\ pranchisco@korea.ac.kr; shimm@korea.ac.kr
}

\section{Extended Abstract}

Biofouling by microorganisms is the most serious problem in membrane processes, as the microorganisms form a biofilm on the membrane surface, resulting in increased chemical and physical resistance. Depositing a metal oxide thin film having antibacterial activity on the membrane surface is an attractive approach to overcome the biofouling problem. However, a very thin and uniform film deposition technique is required to prevent the membrane pore clogging by metal oxide film itself.

Atomic layer deposition (ALD) is a new technology that deposits metal oxides uniformly with nanometer thickness on complicated structures [1], which can overcome the limitations of conventional deposition methods such as chemical vapor deposition and sol-gel method. This study aimed to investigate the antimicrobial effect of zinc oxide ( $\mathrm{ZnO}$ ) thin films formed by ALD and evaluate its applicability to the membrane surface.

A thin $\mathrm{ZnO}$ film was formed with an atomic layer deposition reactor using diethylzinc and deionized water as precursors and oxidants, respectively. Various thin $\mathrm{ZnO}$ films were formed by the number of repetition cycles, and its thickness was analyzed by field-emission scanning electron microscopy. [2] In addition, the permeability was compared between control membrane and modified membrane using a lab-scale filtration unit. The thin $\mathrm{ZnO}$ film was irradiated with UV-A light to induce the photocatalytic reaction, and the antimicrobial effect of the thin $\mathrm{ZnO}$ film was measured through the analysis of viability of Pseudomonas aeruginosa.

The thickness of the $\mathrm{ZnO}$ thin films formed by ALD increased from 10 to $40 \mathrm{~nm}$ in proportion to the number of deposition cycles. As the thickness was much thinner than the pore size of the microfiltration membrane $(200 \mathrm{~nm})$, the $\mathrm{ZnO}$ thin films could not substantially reduced porosity of the membrane. Furthermore, the $\mathrm{ZnO}$ thin films consisted of closely packed nano-sized hexagonal wurtzite crystalline structures which was helpful for the production of reactive oxygen species such as singlet oxygen, hydroxyl radical, and superoxide anion under UV-A irradiation. A filtration test demonstrated that the membrane coated with the $\mathrm{ZnO}$ thin films was more resistant to biofouling than that without the $\mathrm{ZnO}$ films. These results suggest that the membrane coated with $\mathrm{ZnO}$ by ALD could be an effective way in reducing biofouling without hampering filtration performance.

\section{References}

[1] S. M. George, "Atomic layer deposition: an overview," Chem. Rev., vol. 110, pp. 111-131, 2010.

[2] K.-H. Park, "Antibacterial activity of the thin $\mathrm{ZnO}$ film formed by atomic layer deposition under UV-A light," Chem. Eng. J., vol. 328, pp. 988-996, 2017. 